Meta

Journal des traducteurs

Translators' Journal

\title{
La troisième rencontre mondiale de l'AUPELF et les problèmes de traduction
}

\section{Bérke Vardar}

Volume 28, numéro 1, mars 1983

La traduction dans le monde

Translation around the World

URI : https://id.erudit.org/iderudit/004555ar

DOI : https://doi.org/10.7202/004555ar

Aller au sommaire du numéro

Éditeur(s)

Les Presses de l'Université de Montréal

ISSN

0026-0452 (imprimé)

1492-1421 (numérique)

Découvrir la revue

Citer cet article

Vardar, B. (1983). La troisième rencontre mondiale de l'AUPELF et les problèmes de traduction. Meta, 28(1), 93-94. https://doi.org/10.7202/004555ar d'utilisation que vous pouvez consulter en ligne.

https://apropos.erudit.org/fr/usagers/politique-dutilisation/ 


\title{
LA TROISIÈME RENCONTRE MONDIALE DE L'AUPELF ET LES PROBLĖMES DE TRADUCTION
}

\author{
BÉRKE VARDAR
}

La Troisième rencontre mondiale des départements et centres universitaires d'études françaises s'est déroulée du 18 au 24 juillet 1982 à l'Université du Bénin (Lomé, TOGO) et elle a réuni quelque quatre cents participants venus de plus de quatre-vingt-dix pays. Organisée par l'Association des universités partiellement ou entièrement de langue française (AUPELF) avec le concours de l'Agence de coopération culturelle et technique (ACCT), cette troisième rencontre a marqué, par ailleurs, le dixième anniversaire du Comité international des études françaises, créé en 1972, lors de la première rencontre à Québec et complété, en 1977, à Strasbourg, au cours de la deuxième rencontre.

Comme 1'a souligné très pertinemment $\mathbf{R}$. Mallet, président d'honneur de l'AUPELF, «il est nécessaire que les enseignants d'une même langue, à travers les différents cheminements et buts de leur pédagogie, se réunissent pour comparer leurs besoins, leurs méthodes, leurs expériences». En effet, «de telles rencontres permettent de réaliser les plus fructueuses opérations interculturelles. Car il ne s'agit surtout pas de revenir à une uniformatisation des démarches, sous le couvert d'une ratio d'universalisme. Moins encore de favoriser une sorte d'empire linguistique. La langue française voudrait s'honorer d'être un liant et non un lien coercitif ${ }^{1} \gg$.

La nouvelle rencontre de l'AUPELF s'est donc placée sous le signe du français conçu comme un «liant» et un nouvel élan a été donné aux études à travers le thème de réflexion central «Rôles et responsabilités des départements d'études françaises dans leur contexte universitaire, éducatif et culturel, national et international», thème qui a servi à délimiter un terrain d'échange et à ouvrir un forum qui s'est manifesté comme le lieu d'un dialogue entre les théories et les pratiques, ainsi qu'entre les cultures.

Les deux axes principaux autour desquels s'est articulée la Rencontre de Lomé ont été : $I^{\circ}$ les départements d'études françaises dans leur divers contextes; $2^{\circ}$ les études françaises, les différences culturelles et les problèmes de développement. L'un des sous-thèmes proposés à la réflexion des participants portait sur les «départements d'études françaises et les questions de traduction et de terminologie» (sous-thème II.5). L'atelier prévu en II.5a sous l'intitulé «les D.E.F. et les politiques de traduction" a centré la réflexion plus précisément sur les problèmes de traduction. J.C. Corbeil a rappelé, dans son rapport in-

1. «Un liant plus qu'un lien», le Monde, 20 juillet 1982. 
troductif, que deux conceptions de la traduction coexistent de nos jours dans les universités : la traduction «littéraire» et la traduction «professionnelle» qui va en se généralisant, ce qui est de nature à modifier substantiellement les programmes d'études. D'autres centres d'intérêt ont également retenu l'attention du rapporteur: de ce nombre sont la traduction considérée comme moyen de connaissance des autres par soi et de soi par les autres, et les orientations de la coopération internationale en matière de traduction.

Les travaux de l'atelier prévu en II.5a ont été animés par J.-C. Gémar, B. Vardar et Youssif Elias, et ont porté essentiellement sur quatre centres d'intérêt majeurs : la traduction pédagogique, l'interprétation, la traductologie et la traduction littéraire. Les communications inscrites dans le cadre de cet atelier ont été discutées à partir des résumés présentés par leurs auteurs. En ce qui concerne le premier centre d'intérêt, J.-C. Gémar a mis l'accent sur le rôle de la traduction dans la défense et l'illustration de la langue française au Québec; J.-G. Trépanier a traité des problèmes de traduction relevant de l'enseignement du français dans un contexte de multilinguisme; Youssif Elias a abordé la traduction littéraire telle qu'elle doit être utilisée au sein des départements d'études françaises; B. Vardar a évoqué l'importance de la traduction et de la terminologie dans un cursus d'études françaises et $\mathrm{B}$. Wilmet a souligné la nécessité de la traduction des didacticiels en français. Le deuxième centre d'intérêt de l'atelier a été représenté par $\mathrm{A}$. Kunte qui a traité du rôle de l'interprète et de la nature de son travail, en prenant notamment en considération les séances au sommet. La traductologie ou théorie de la traduction a fait l'objet de l'exposé de C. Tatilon qui a évoqué les problèmes posés par l'extraction de l'information pertinente dans l'activité traduisante. Quant à T. Inal, il a abordé la traduction littéraire par le biais du plaisir esthétique et a souligné notamment l'importance de la traduction dans l'enrichissement du bagage culturel et la formation de l'esprit de l'étudiant, ce qui lui a permis de rejoindre, par là même, le premier centre d'intérêt de l'atelier. Par ailleurs plusieurs expériences intéressantes ont été évoquées par les intervenants. Le «portrait d'un centre d'activités langagières en cours de création» à Imo State University, Nigeria, présenté par A. Ihenacho a surtout retenu l'attention des participants. Au cours des débats, on a insisté sur la nécessité de bien distinguer la traduction pédagogique qui relève de la didactique, de la pédagogie de la traduction qui est orientée vers la formation professionnelle d'élèves-traducteurs, et la traduction utilitaire de la traduction littéraire. Parmi les recommandations formulées par les intervenants, celle prévoyant la communication de toute la documentation nécessaire aux personnes chargées de la création d'un centre d'interprètes et de traducteurs (cf. supra) ainsi que celle souhaitant l'intensification des échanges d'idées et d'informations par l'intermédiaire de l'AUPELF ont été particulièrement retenues dans le rapport général de synthèse.

La Rencontre de Lomé a été donc, aussi bien en ce qui concerne la traduction que pour ce qui est des autres activités que comportent les études françaises, la confirmation que les départements d'études françaises sont «un lieu de contact» et qu'ils se trouvent, par là même, «ancrés au coeur de 1'interculturel», comme l'a affirmé, dans le rapport général de synthèse, le professeur M. Tétu, président du Comité international des études françaises. 